\title{
Multi-Criteria Fuzzy-Based Decision Making Algorithm to Optimize the VHO Performance in Hetnets
}

\section{A. Prithiviraj ${ }^{1, *}$, A. Maheswari ${ }^{2}$, D. Balamurugan ${ }^{1}$, Vinayakumar Ravi ${ }^{3}$, Moez Krichen ${ }^{4,5}$, Roobaea Alroobaea ${ }^{6}$, Saeed Rubaiee ${ }^{7}$ and Sankar Sennan}

\author{
${ }^{1}$ Department of CSE, Sona College of Technology, Salem, 636005, India \\ ${ }^{2}$ Department of CSE, Dr. M. G. R. Educational and Research Institute, Chennai, 600095, India \\ ${ }^{3}$ Center for Artificial Intelligence, Prince Mohammad Bin Fahd University, Khobar, Saudi Arabia \\ ${ }^{4}$ Faculty of CSIT, Al-Baha University, Saudi Arabia \\ ${ }^{5}$ ReDCAD laboratory, University of Sfax, Tunisia \\ ${ }^{6}$ Department of Computer Science, College of Computers and Information Technology, Taif University, \\ Taif, 21944, Saudi Arabia \\ ${ }^{7}$ Department of Industrial and Systems Engineering, College of Engineering, University of Jeddah, Jaddah, Saudi Arabia \\ *Corresponding Author: A. Prithiviraj. Email: prithivi@sonatech.ac.in \\ Received: 14 November 2020; Accepted: 17 April 2021
}

\begin{abstract}
Despite the seemingly exponential growth of mobile and wireless communication, this same technology aims to offer uninterrupted access to different wireless systems like Radio Communication, Bluetooth, and Wi-Fi to achieve better network connection which in turn gives the best quality of service (QoS). Many analysts have established many handover decision systems (HDS) to enable assured continuous mobility between various radio access technologies. Unbroken mobility is one of the most significant problems considered in wireless communication networks. Each application needs a distinct QoS, so the network choice may shift appropriately. To achieve this objective and to choose the finest networks, it is important to select a best decision making algorithm that chooses the most effective network for every application that the user requires, dependent on QoS measures. Therefore, the main goal of the proposed system is to provide an enhanced vertical handover (VHO) decision making program by using a Multi-Criteria Fuzzy-Based algorithm to choose the best network. Enhanced Multi-Criteria algorithms and a Fuzzy-Based algorithm is implemented successfully for optimal network selection and also to minimize the probability of false handover. Furthermore, a double packet buffer is utilized to decrease the packet loss by $1.5 \%$ and to reduce the number of handovers up to $50 \%$ compared to the existing systems. In addition, the network setup has an optimized mobility management system to supervise the movement of the mobile nodes.
\end{abstract}

Keywords: Vertical handover; mobility management; handover decision systems; multi-criteria; fuzzy logic; QoS; false handover 


\section{Introduction}

Providing widespread and consistent availability to mobile terminals while moving among heterogeneous wireless environments is probably the greatest challenge, analysts have faced for numerous years. Therefore, in the heterogeneous network environment, the primary focus of this challenge is to make an appropriate and clever choice for handover. In an active session, it is necessary for the Mobile Node (MN) to accomplish the horizontal handover (HHO) or VHO when it moves within a heterogeneous wireless network (Hetnets), to maintain its connection. Altogether, an intellectual Handover Decision Systems (HDS) is needed to choose the finest network for the handover. One of the important factors in the decision making is the mobility speed of the mobile terminal, which need to be considered in the decision making technique to reduce the false handovers that generally contribute to the number of unnecessary handovers.

The main emphasis of next generation mobile networks is on flawlessly connecting the prevailing wireless technologies like Wi-Fi, 2G, 3G, 4G, Wi-Max, and WLAN into an all-IP based heterogeneous network. Perhaps a vital challenge for next generation wireless networks is to bring together these various wireless network technologies into one all IP-based. These different network technologies ought to be synchronized in an enhanced manner with the main aim being to provide the services requested by the user along with the user QoS requirements. One of the most perplexing problems among the networks is VHO in a heterogeneous network environment. Heterogeneous wireless networks are described as the framework encompassing various radio based communication networks such as Wireless Fidelity (Wi-Fi), Universal Mobile Telecommunications System (UMTS), Worldwide Interoperability for Microwave Access (Wi-MAX), Global System for Mobile Communications (GSM), and Wireless Local Area Network (WLAN) [1]. Every wireless technology has its own special features in terms of service cost, bandwidth, data rate, latency, cell size, received signal strength, network occupancy and coverage.

Handover is a technique in mobile communications wherein a mobile device is in a linked information session starting from one cell site and moving onto the next [2]. VHO takes place when the handover process is among different radio access technologies whereas HHO takes place among the same radio technology or the same network, as represented in Fig. 1. Consistency in handover is achieved by having a low handover latency, seamless connectivity, and negligible packet loss. The time delay between the mobile node receiving the final data packet in the previous serving network and receiving the first data packet in the new serving network is termed handover latency. Commonly, handover latency refers to the time taken by the mobile node to be committed to the new ideal network.

False handover [3] occurs due to improper choice of enhanced network during handover between the previous serving network and the new network. The VHO process can be categorized into three steps, namely handover initiation, handover decision, and handover execution [4]. During the handover initiation stage, various data are considered concerning the network from various layers such as Link Layer, Application Layer and Transport Layer. Data such as cost, bandwidth, link speed, RSS, power, jitter, user preferences, throughput and network subscription are provided by the layers. The proposed framework is evaluated using two datasets and was compared with recent deep learning models. Various visualization methods were also employed to understand the characteristics of the dataset and to visualize the embedding features [5]. The mobile node makes decisions on whether to continue the connection with the present wireless network or else to change to another network during the handoff decision process. The decision depends on numerous constraints of the network that were gathered in the handover initiation stage. The decision making is carried out by using various decision making algorithms such as 
traditional, function-based, context-aware, user-centric, multiple attributes decision making, neural networks, and Fuzzy logic algorithm. In the paper, a multi-criteria fuzzy-based handover decision system design for selecting the best network is proposed. During the handover execution stage, existing connections should be moved to the new network in an uninterrupted manner.

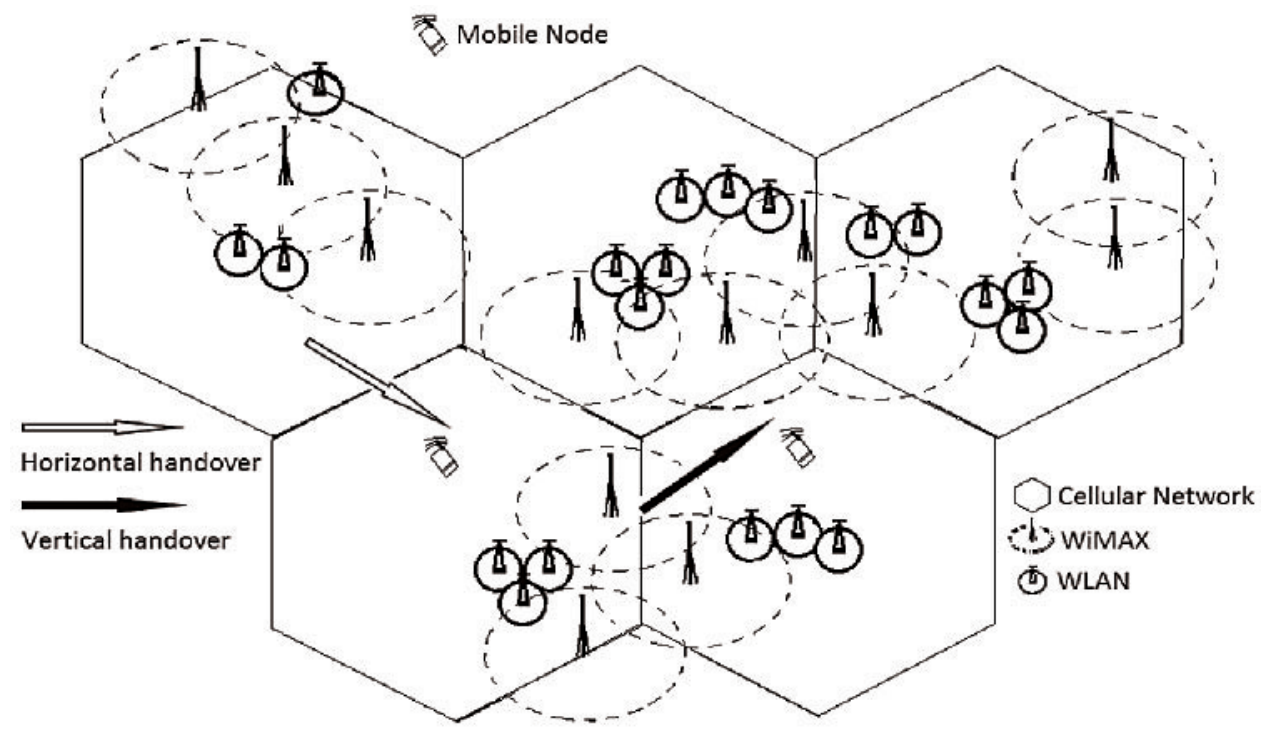

Figure 1: Sample heterogeneous wireless networks environment

The rest of the paper is organized as follows. Section 2 reviews the different state-of-theart multi attribute decision making and fuzzy based algorithms used in VHO processes for the selection of the appropriate network to perform handover. Section 3 presents the implementation architecture of the mobility management mechanisms and the proposed methodologies based on multi-criteria and fuzzy based decision making algorithms. Section 4 describes the experimental setup and discusses the results obtained from the proposed algorithms over the existing approaches. Section 5 provides the conclusion of the research and future work.

\section{Related Works}

\subsection{An Overview of $\mathrm{VHO}$}

The paper by Bhuvaneswari et al. [6] describes the summary of handover process, handover types, and classification of VHO, parameters requirements, and comparison table. The different types of decision making algorithms described in the proposal are (1) context-aware, (2) functionbased decision making, (3) Fuzzy logic and neural networks, (4) Multiple attributes decision making algorithm (MADM), (5) Traditional and (6) User-Centric. The paper explains the VHO decision making algorithms in detail. Additionally, several methods have been proposed by Amandeep Singh Sandhu in the literature survey [7], although, there is no productive technique or standard to satisfy both network necessities and user or client regarding QoS.

\subsection{Algorithms Based on MADM}

Various papers that explain MADM algorithms are discussed by Santhi et al. [8] and the comparison between them is made to provide handover solutions. The various common features 
of MADM problems such as Alternatives, Attribute weights, Decision matrix, Multiple attributes and Normalization are clarified. The proposal by Mahardhika et al. [9] is used to calculate the corresponding weights of the criteria. Well-known algorithms such as Analytic Hierarchy Process (AHP) and Analytic Network Process (ANP) are used in this proposal. The most dedicated algorithms for categorizing candidate networks are the Gray Relational Analysis (GRA), Multiplicative Exponential Weighting (MEW), Technique for Order Preference by Similarity to the Ideal Solution (TOPSIS), Simple Additive Weighting (SAW), and Vlse Kriterijumska Optimizacija I Kompromisno Resenje (VIKOR), the optimization of conflicting multi-criteria to achieve an acceptable compromise solution, described in the proposal by Baghla et al. [10]. These algorithms were analyzed for evaluating the number of handovers, performance of handover latency and optimum network selection. The attributes such as cost per byte, delay, available bandwidth, jitter, security, and packet loss are calculated for several networks using MADM algorithms. The proposed algorithm by Abdullah et al. [11] and another proposal by Abdullah et al. [12] incorporated three differing VHO decision making algorithms such as: Equal weight, Mobile weight, and Network weight. In the proposed hetnets, the output of a layer is added to the next input and then served in the same layer. The output controls the information of signal with respect to the time [13]. Finally the simulation checks the handover decision making algorithm by calculating the network weightage, generated with exceptional outputs. The results are compared with traditional network decision algorithm looking at failure of handover and number of handover possibilities.

\subsection{Algorithms Based on Fuzzy}

The proposal by Sharma et al. [14] which describes the significant use of fuzzy logic related algorithms in the heterogeneous wireless environment is highlighted by the author. Sharma's proposal also focuses on routing in mobile Ad-hoc networks, application of fuzzy logic in cognitive radio, decision making in heterogeneous wireless environment, wireless sensor networks etc. The performance of different parameters in wireless environments can be improved using fuzzy logic algorithms. Fuzzy logic is theoretically easy to follow and pliable. Furthermore, the mathematical ideas behind fuzzy reasoning are extremely basic. Fuzzy inference is the process of producing a resultant value based on a fuzzy rule or IF-THEN rule with the input parameters mapped to the fuzzy engine's input. Such operation is executed by the fuzzy inference system.

The fuzzy IF-THEN rules that exist in the rule base are: It consists of the defined membership functions of the fuzzy sets. The inference operations take place in the decision making part of the rules. A fuzzification interface of the fuzzy rule is used to propagate the fragile inputs into the degrees of matches with linguistic values. Then, a defuzzification interface is used to transform the fuzzy results into crispy output.

\section{Proposed System}

Heterogeneous wireless networks can provide widespread and seamless connectivity for any types of service user. Heterogeneous wireless networks are well-defined as the structure consisting of numerous radio accessing technologies such as Wireless Fidelity (Wi-Fi), Universal Mobile Telecommunications System (UMTS), Wireless Local Area Network (WLAN) and Worldwide Interoperability for Microwave Access (WI-MAX). Each wireless technology has its own distinct characteristics in terms of bandwidth, coverage, data rate, network occupancy and RSS. Throughout the movement of the $\mathrm{MN}$ attached to the home network, the $\mathrm{MN}$ should have the best connectivity with the finest network. The main goal is to connect with the best network for the mobile node moving across networks but the selection of network may vary depending on the 
QoS requirement of each application running on the mobile node. The optimal decision making algorithm is mandatory in order to meet the user service requirements based on the QoS criteria with the selected best network. This proposed system is about enhanced VHO decision making by using a Multi-criteria Fuzzy-Based algorithm to select the best network. Enhanced MultiCriteria algorithms and Fuzzy Logic based algorithms are used effectively to select the optimal network and also to reduce the probability of false handover. Additionally, a packet buffer is used to reduce the packet loss in the mobility management mechanism. An efficient mobility management mechanism is also provided in the networking environment and this also has network controls for concerned mobile nodes which are in mobility. To assess the proposal on the Multicriteria and Fuzzy based Decision making algorithm, the assessment is based on network selection performance, for which the Network simulator tools are used to develop the environment and experiment the system.

\subsection{Mobility Management}

The proposal aims to provide an optimal solution for seamless connection by intelligent VHO algorithms. The architecture model to represent the handover among UMTS, WLAN, Wi-Fi, and WI-MAX in a heterogeneous environment is shown in Fig. 2. The proposal mainly reduces computation time and packet loss.

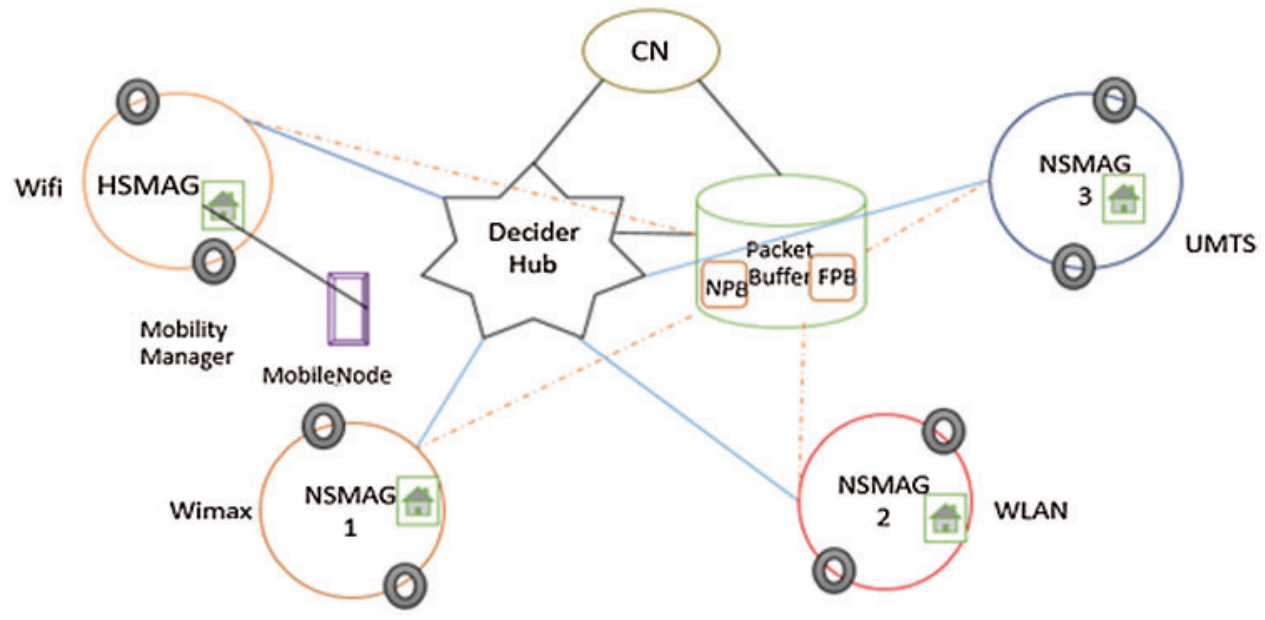

Figure 2: Architecture of the proposed mobility management mechanism

The Decider hub (DH) in the model, which is considered as the spine of the system is where its various connections to different networks in the scenario are maintained. When the $\mathrm{MN}$ is idle, a DH acts as a centralized server performing the decision making for handover in selecting the best network and buffers for the ongoing packets. The buffering mechanism is taken care of by a packet buffer which is categorized as New Packet Buffer (NPB) and Forward Packet Buffer (FPB). For monitoring the mobility of nodes, few mobility managers (MM) are present in each network depending upon the coverage area. 


\subsection{Phases of Handover}

\subsubsection{Handover Initiation Phase}

At initial state, the MN is attached to the Home Serving Media Access Gateway (HSMAG). HSMAG is the Media Access Gateway (MAG) of the home network by which the MN and Correspondent node $(\mathrm{CN})$ interconnects. When $\mathrm{MN}$ enters its coverage area, it is the responsibility of MAG to authenticate it. The mobility of nodes is traced by the mobility managers and the location of the $\mathrm{MN}$ is passed to MAG and $\mathrm{DH}$. The number of MMs might differ, and are placed in such a way that, they cover the node's action in MM's range. For reliable transmission to optimal networks, the DH maintains the routing table [15].

The Mobility Manager has the signal strength values i.e., RSS and the distance value to predict the MN that will become detached from the Home Network (HN) in the near future. When the $\mathrm{MN}$ is moving out of range, the signal strength reduces, and distance is increased. The continuous change in network behavior and rapid evolution of attacks makes it necessary to evaluate various datasets which have been generated over the years through static and dynamic approaches [16]. Handover initiation is intimated to the DH by these two values. Then the DH broadcasts all networks in the heterogeneous environment about the MN's idle state with the MN's profile. Significant information gathered is used by the next phase, the decision phase, thus helping to predict the destination network accurately.

\subsubsection{Handover Decision Phase}

Usually the DH has certain algorithms to be used for selecting the best networks and it is necessary for the mobile node to be connected to the network that the DH chooses, in its idle state. The $\mathrm{DH}$ is equipped with a decision making algorithm with its required inputs gathered in the initiation phase. Data transfer from the correspondent node to the destination node occurs via the DH and packet buffer. The DH transfers the data packets to the appropriate network MAG to which the Mobile Node is attached. The DH comprises enhanced multi criteria algorithms such as Technique for Order of Preference by Ranking to Exact Solution (TOPRES), Ranking Algorithm for Multicriteria Optimization and Compromisation (RAMOC) and Fuzzy based decision making algorithm. The destination network is chosen based on the majority of the result produced by these algorithms. The multicriteria-fuzzy technique enhances the intelligence of the handover decision system by reducing the algorithm execution time and the packet loss.

\subsubsection{Handover Execution Phase}

The Decision Phase predicts the best network to attach to. Based on this the MN establishes its connection path with the new serving media access gateway (NSMAG). The DH updates its routing table information for the $\mathrm{CN}$, to transmit data to the new network. The packets that are buffered using packet buffers when the $\mathrm{MN}$ is in idle state are transferred to the newly created connection to reduce loss of packets [17]. This is done prior to connection to the new network and then it decides its route for packet transfer. To provide effective seamless connection, the mobility management is also taken care of by the execution phase of handover. The issues in handover like packet loss have been addressed.

The pseudo-code of the proposed model is given below, 


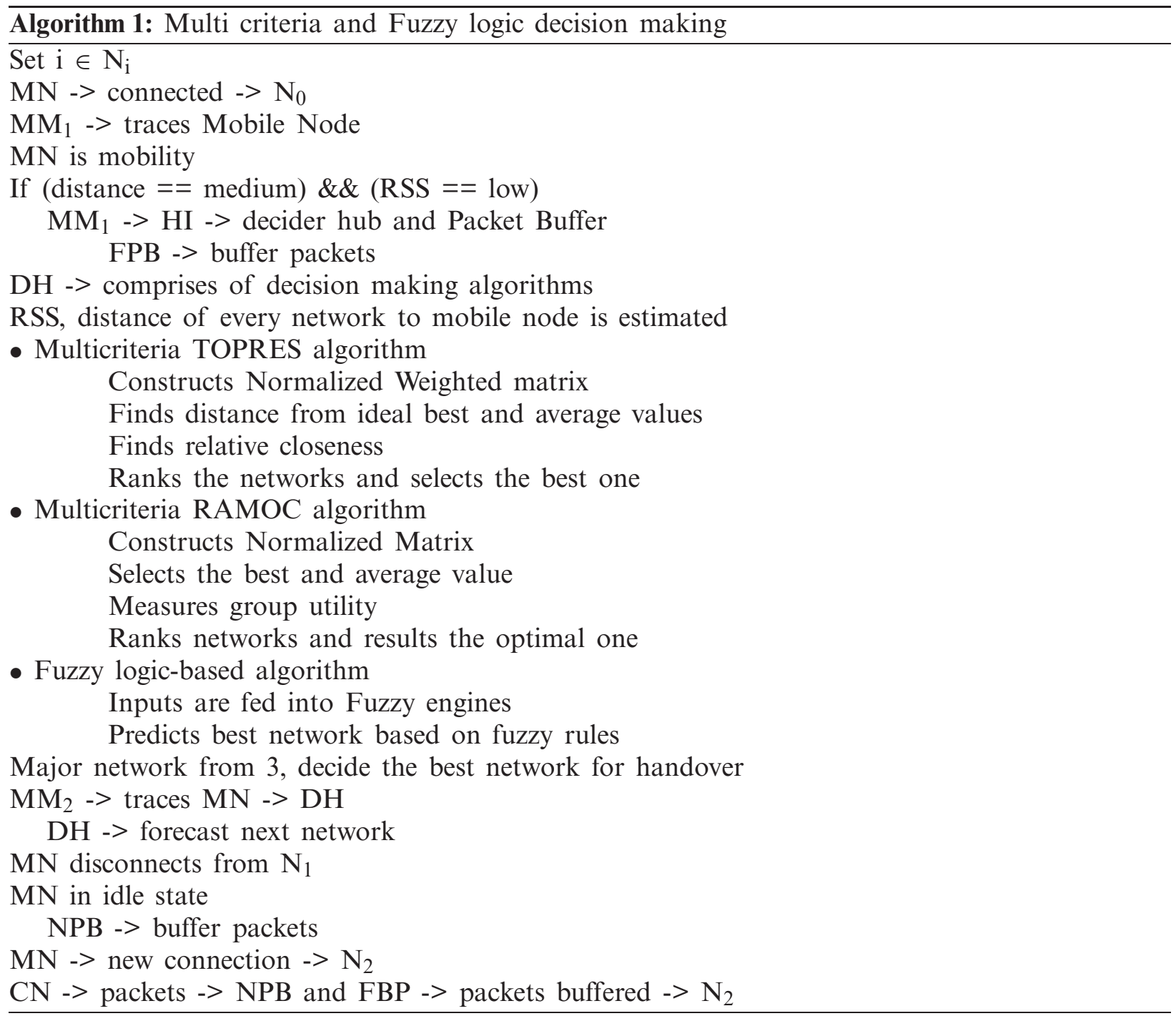

\subsection{Message Signaling Flow}

The steps involved in the proposed system as represented in Fig. 3 are explained below,

Step 1: In order to access the applications and their services in the internet worldwide, the mobile node will be connected to a network. The authentication by the HSMAG is compulsory for a node to be attached to the home network as an authenticated MN involved in the data transfer to the MN. It also allocates an ID to the MN. Further, the MAG sends the DH, a proxy bind update message as well as the profile of the MN. To locate the destination path, the MN checks within the $\mathrm{DH}$.

Step 2: A proxy acknowledgement message is received as response from $\mathrm{DH}$, thus between them, the channel is made. The Home Network is the network to which the MN is attached, and Home Serving MAG is the MAG for the MN.

Step 3: Communication takes place between the $\mathrm{CN}$ and the mobile node $(\mathrm{MN})$ via the $\mathrm{DH}$ and the HSMAG. For the packets to reach the right destination network, the DH Routing table is 
used. As the HSMAG needs to find out the destination node that is waiting for the data, the data packet is prefixed with MN-ID and transmission of data between MN and CN occurs successfully.

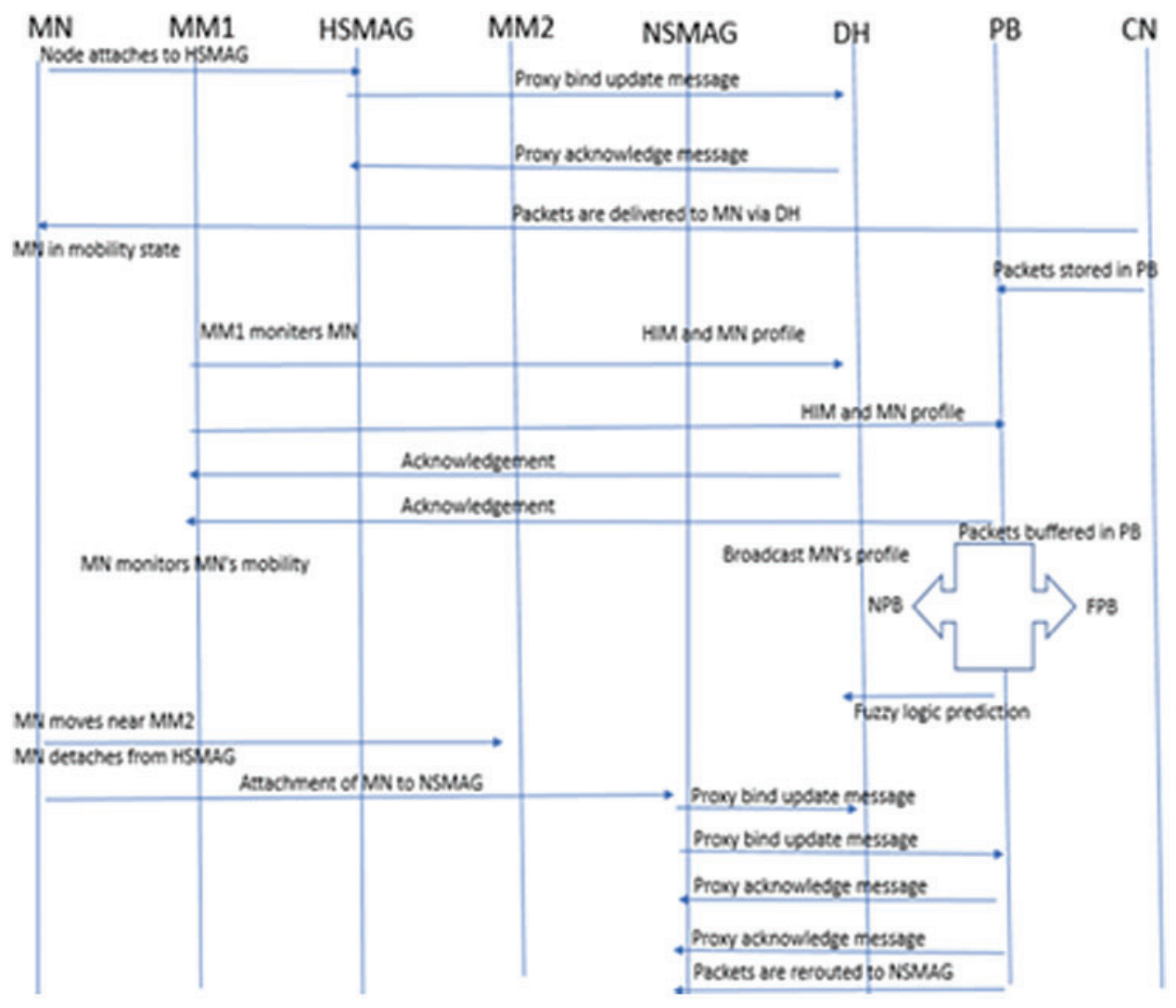

Figure 3: Message signaling flow

Step 4: The MN keeps going away from the current network and finally completely goes out of range of the Home Network.

Step 5: Prior to the complete disconnection from a network, the mobility manager placed at certain areas based on coverage area, will track the mobility of MN. After examining the ranges of distance of $\mathrm{HN}, \mathrm{MN}$, and RSS, the Mobility manager-1 informs the MAG, and thereafter MAG informs DH and packet buffer about the connection loss that is going to occur.

Step 6: DH receives Handover Initiation Message (HIM), MN's profile and an acknowledgement message for initiation is sent from the packet buffer.

Step 7: The DH, broadcast the profile of the MN to all the neighboring networks available within the surroundings.

Step 8: After HIM is received, packet loss is avoided by using a double buffer mechanism. In this mechanism, packets are buffered by packet buffer from $\mathrm{CN}$ at the time of the $\mathrm{MN}$ entering into the handover session.

Step 9: In the meantime, the MN gets into the handover period as it is entirely detached from the Home Network. Between the isolated Mobile Node and the CN, the link for communication is destroyed. 
Step 10: MN's location is periodically monitored, and its migration is looked after by DH. DH is binded with Multicriteria Fuzzy logic-based handover decision making to decide a new network (NAN) and get its specifications.

Step 11: Monitoring the movement of the Mobile Node and measuring data rate, bandwidth, coverage, RSS, and network occupancy value when the MN is idle in the participating network and in movement of the MN is carried out by the MM of other networks such as MM2. The $n$ the acquired values are directed to $\mathrm{DH}$ for selecting the finest network so that the mobile node can get attached to the best available network.

Step 12: The node is authenticated by the New network MAG of NAN. A channel of communication is generated among Mobile Node and Corresponding Node through PB, DH and NSMAG in the stated Steps 1 to 3.

Step 13: The routing table of the DH is updated for accurate data transfer between the Mobile Node and Corresponding Node by optimal routing of NAN.

Step 14: PB transmits the buffered packets until the best route is created [18]. Double buffering mechanism enables the data packets to reach the correct destination in an exact sequence as the packet is prefixed with the sequence number thus minimizing packet loss.

\subsection{Buffering Mechanism}

When the mobile node ( $\mathrm{MN})$ is in idle state, there is no transfer of data packets between Mobile Node and Corresponding Node. Until an appropriate network is found, the packet buffer buffers the data packets and they are re-transmitted once a new network connection is established. The packet buffer stores the data packets with sequence numbers. In this mechanism, two buffers, New Packet Buffer and Forward Packet Buffer, are used for buffering packets. The stages involved in the buffering process are listed below,

Step 1: In a regular case, $\mathrm{CN}$ transmits the data packets to the home network over the DH. When the DH and packet buffer receive HIM, the DH informs the Forward Packet Buffer of the PB to start buffering the packets with sequence IDs and forward them to HSMAG.

Step 2: While the $\mathrm{MN}$ is detached from home network, the $\mathrm{DH}$ then intimates to the New Packet Buffer to buffer the data packets with sequence number from which the MN is disconnected from the home network.

Step 3: DH manages the sequence numbers. By prior transmission of packets across buffers, $\mathrm{MN}$ receives packets without loss in a sequence at the time when a new channel is established.

\subsection{Decision Making Algorithms}

As the multicriteria algorithm when combined with Fuzzy logic-based algorithms improve network performance by reducing the delay and packet loss, so TOPRES and RAMOC derived from Multicriteria algorithm and Fuzzy-based algorithms are used and described below:

\subsubsection{Multi-Criteria Decision Making Algorithm}

Multi-criteria decision making helps in making ideal VHO decisions as there are various target network options. Likewise, multicriteria decision making delivers high throughput, reduced packet loss, flexibility and choosing the best network by considering more criteria.

\section{Normalization}

The five important normalization techniques, listed below are compared and analyzed, 
- Vector Normalization

- Linear Max-Min Normalization

- Linear Sum based Normalization

- Linear Max Normalization

- Gaussian Normalization

By considering various normalization techniques [19] it is noticed that the Linear Sum based Normalization technique attains less Space Complexity and computation time. Hence Linear Sum based Normalization is used in our methods rather than traditional methods to improve performance and efficiency.

Technique For Order Of Preference By Ranking To Exact Solution (TOPRES)

Steps involved:

Step 1: Construction of the normalized Decision Matrix.

Multi-criteria decision making is represented in matrix form. The decision matrix denoted as $A$ is an $(m * n)$ matrix where $i^{\text {th }}$ row refers to the network and $\mathrm{j}^{\text {th }}$ column refers to a characteristic or an attribute, (for $\mathrm{i}=1,2, \ldots, \mathrm{m}$ and $\mathrm{j}=1,2, \ldots, \mathrm{n}$ ).

$$
\begin{aligned}
& C_{1} C_{2} \ldots C_{n} \\
& \begin{array}{llllll}
A_{1} & a_{11} & a_{12} & \ldots & a_{1 n}
\end{array} \\
& \begin{array}{lllll}
A_{2} & a_{21} & a_{22} & \ldots & a_{2 n}
\end{array}
\end{aligned}
$$

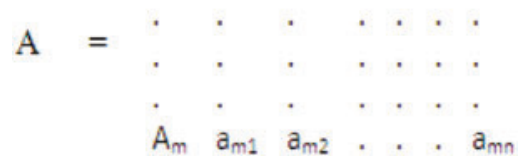

To change the dimensional characteristics into non-dimensional ones, the equation is used based on its type,

For beneficial attributes,

$\mathrm{r}_{\mathrm{ij}}=\frac{\mathrm{a}_{\mathrm{ij}}}{\sum_{i=1}^{m} \mathrm{a}_{\mathrm{ij}}}, \quad \mathrm{i}=1, \ldots, m, \quad \mathrm{j}=1, \ldots, n$

For non-beneficial attributes,

$\mathrm{r}_{\mathrm{ij}}=\frac{\mathrm{a}_{\mathrm{ij}}}{\sum_{i=1}^{m}\left(1 / \mathrm{a}_{\mathrm{ij}}\right)}, \quad \mathrm{i}=1, \ldots, \mathrm{m}, \quad \mathrm{j}=1, \ldots, \mathrm{n}$

where,

$\mathrm{a}_{\mathrm{ij}}$ denotes the score of networks $\mathrm{i}$ with respect to attribute $\mathrm{j}$.

$\mathrm{m}$ denotes the various alternatives (Wi-Fi, WiMAX, UMTS and WLAN).

$\mathrm{n}$ denotes the criteria/attributes (Data rate, RSS, Bandwidth, Coverage, \& network occupancy).

Step 2: Construction of weighted normalized decision matrix. Each column of the normalized decision matrix is multiplied by their criteria weights as given below,

By using the equation,

$\mathrm{V}_{\mathrm{ij}}=\mathrm{W}_{\mathrm{j}} * \mathrm{r}_{(\mathrm{ij})}$

where $\mathrm{Wj}$ denotes the weight of the $j$ th criteria. 
Step 3: Determine the ideal best and the ideal average alternatives. They are given by,

$$
\begin{aligned}
& \mathrm{V}^{+}=\left\{\mathrm{V}_{1}^{+}, \mathrm{V}_{2}^{+}, \ldots, \mathrm{Vn}^{+}\right\}=\underset{i}{\max }(\mathrm{Vij}) \\
& \mathrm{V}^{\mathrm{avg}}=\mathrm{V}_{1}^{\mathrm{avg}}, \mathrm{V}_{2}^{\mathrm{avg}}, \ldots, \mathrm{V}_{\mathrm{n}}^{\mathrm{avg}}=\underset{i}{\operatorname{avg}}\left(\mathrm{V}_{\mathrm{ij}}\right)
\end{aligned}
$$

Step 4: Calculating distance from ideal best and average values.

$$
\begin{aligned}
& \mathrm{Si}^{+}=\sqrt{\left(V_{i 1}-V_{j}^{+}\right)^{2}+\left(V_{i 2}-V_{j}^{+}\right)^{2}+\ldots+\left(V_{i n}-V_{n}^{+}\right)^{2}} \\
& \mathrm{Si}^{\text {avg }}=\sqrt{\left(V_{i 1}-V_{j}^{a v g}\right)^{2}+\left(V_{i 2}-V_{j}^{a v g}\right)^{2}+\ldots+\left(V_{i n}-V_{n}^{a v g}\right)^{2}}
\end{aligned}
$$

Step 5: Calculation of relative closeness to ideal solution is defined by

$P_{i}=\frac{S_{i}^{a v g}}{S_{i}^{+}-S_{i}^{a v g}}, \quad(i=1,2, \ldots, m)$

Hetnets are ranked from maximum value as Rank 1, then Rank 2 and so on. So, by this ranking TOPRES selects the best network.

Ranking Algorithm For Multicriteria Optimization And Compromization (RAMOC)

\section{Steps involved:}

Step 1: Construction of the Normalized Decision Matrix.

Here, the decision matrix, $D=[a i j]_{m \times n}$ is constructed for networks with its attributes as in Step 1 in TOPRES. Where $i$ th row signifies the various networks, $j$ th column signifies characteristic or attribute. As described, the Linear-Sum based normalization is carried out.

The equation used is based on its type,

For beneficial attributes,

$\mathrm{r}_{\mathrm{ij}}=\frac{\mathrm{a}_{\mathrm{ij}}}{\sum_{i=1}^{m} \mathrm{a}_{\mathrm{ij}}}, \quad \mathrm{i}=1, \ldots, m, \quad \mathrm{j}=1, \ldots, n$

For non-beneficial attributes,

$\mathrm{r}_{\mathrm{ij}}=\frac{\mathrm{a}_{\mathrm{ij}}}{\sum_{i=1}^{m}\left(1 / \mathrm{a}_{\mathrm{ij}}\right)}, \quad \mathrm{i}=1, \ldots, m, \quad \mathrm{j}=1, \ldots, n$

where,

$a_{i j}$ denotes the score of networks $i$ with respect to attribute $j$

$\mathrm{m}$ denotes the various alternatives (Wi-Fi, WiMAX, UMTS \& WLAN).

$\mathrm{n}$ denotes the criteria/attributes (Datarate, RSS, Bandwidth, Coverage, \& network occupancy).

Step 2: Selection of best and average attribute value.

Based on the values in the decision matrix and whether the values falls under beneficial and non-beneficial category of the attribute the best value $\left(f_{j}^{*}\right)$ and average $\left(f_{j}^{a v g}\right)$ values are selected. 
For the beneficial category, the maximum value is the best value and for the non-beneficial category, the minimum is the best value.

\section{Step 3: Measure of group utility.}

Based on the type, the attribute is categorized as a beneficial or non-beneficial attribute. Every attribute of a network is given a weight $(0<w j<1)$ which indicates its priority or importance. $\mathrm{Si}$ is the measure of group utility [20] i.e., the sum of weighted normalizations of entire attributes in a certain network.

$S i=\sum_{j=1}^{n}\left(f_{j}^{*}-a_{i j}\right) /\left(f_{j}^{*}-f_{j}^{a v g}\right)$

Step 4: Individual regret for an alternative.

Then, the extreme value of each network is taken and denoted as $\mathrm{R}$.

$\mathrm{R}_{i}=\operatorname{Max}\left(S_{i}\right)$ for each network

where, $R_{i}$ is known as individual regret for an alternative.

Step 5: Ranking the networks.

The average of $\mathrm{S} i$ and $\mathrm{R} i$ is calculated and based on the deviation of the individual result of $\mathrm{S} i$ and $\mathrm{R} i$, the networks are ranked as $\mathrm{S} i$ Rank and $\mathrm{R} i$ Rank. Finally, an overall rank from these two is derived and RAMOC selects the optimal network.

\section{Fuzzy-Based Handover Decision Systems}

Fuzzy logic is widely used as an instrument to improve the knowledge of decision-making mechanisms in various regions. Many efforts are made to safeguard the QoS, as the interest for real-time applications is continually growing. But the fuzzy membership functions are fixed and the design structure of most of the fuzzy logic-based handover decision algorithms is monolithic. The two drawbacks of such designs are: when the count of input parameters grows, the time of execution of algorithm increases, and with various kinds of traffic or services, performance of the network selection decreases. To overcome these issues, several engines should be utilized in handover decision algorithms. Multiple fuzzy engines are designed to choose the best network for handover. Fig. 4 describes the fuzzy inference engine.

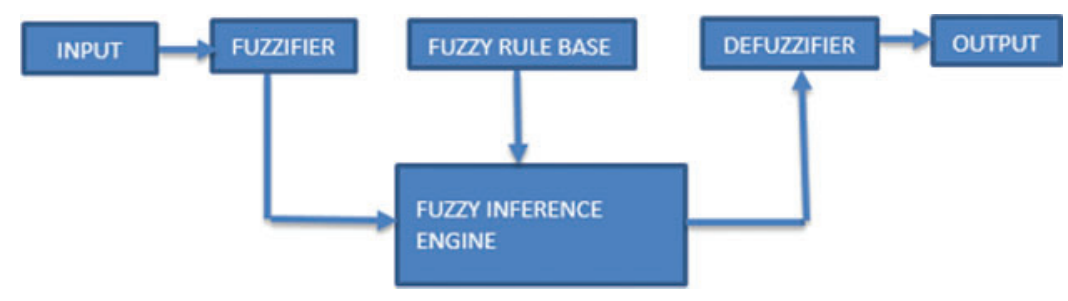

Figure 4: Fuzzy inference engine

\section{Multi-Criteria Fuzzy-Based Handover Decision System Design}

The proposed multi-criteria fuzzy based handover decision system design is shown in Fig. 5, the proposed HDS which comprises four fuzzy engines [21], namely a Degree of Satisfaction (DS) engine, an Aggregated QoS (AQ) engine, a Network QoS (NQ) engine and a Mobility Engine 
(ME). In order to make an intellectual and comprehensive decision during the handoff process, five decision constraints like RSS, Band Width (BW), Data Rate (DR), Network Occupancy (NO), Coverage $(\mathrm{CO})$ are taken into consideration. Every fuzzy engine operates with a specific collection of decision attributes. The two inputs, BW and DR, are fed into the AQ fuzzy engine to provide the A (value) thereby measuring the QoS of every network. NQ fuzzy engine provides the A (value) and the ME fuzzy engine provides the value of CO and NO. The DS fuzzy engine is fed with the output of the NQ and ME fuzzy engines. For every candidate network, the DS fuzzy engine estimates the resulting $\mathrm{D}$ value. Normally the handover decision is made based on the best network available among heterogeneous wireless networks. The best candidate network should have good coverage, bandwidth, received signal strength, data rate and network occupancy. Fuzzy rules are framed based on these parameters.

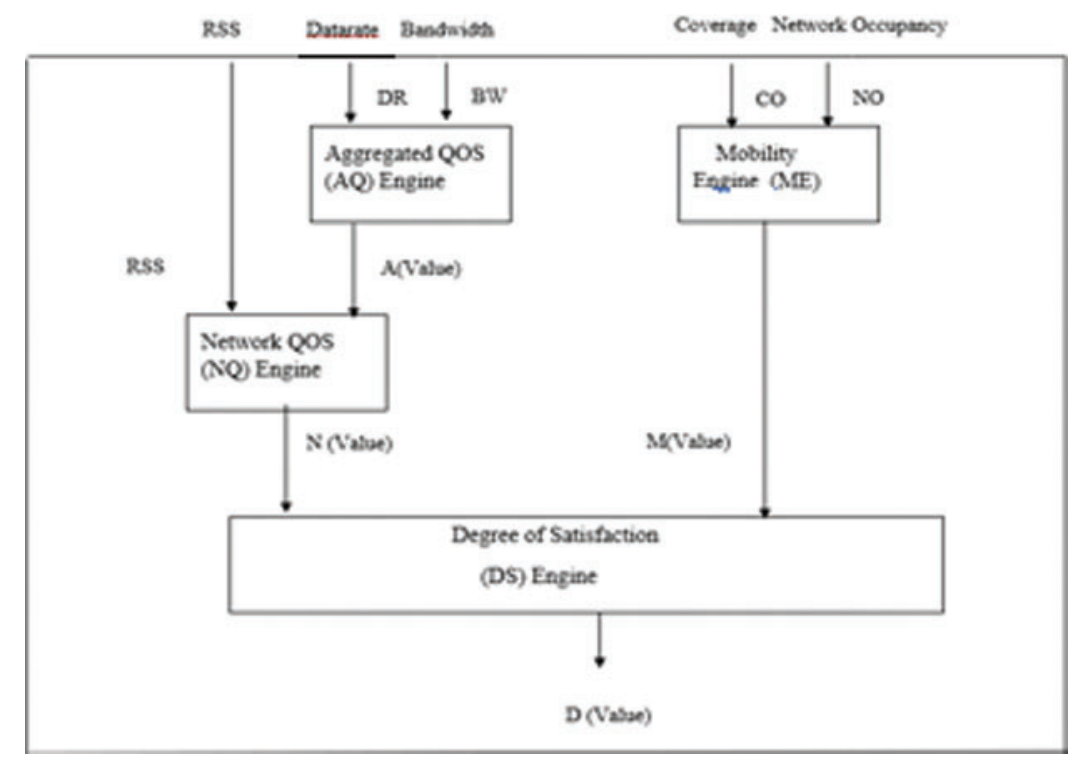

Figure 5: Fuzzy based HDS design

\section{Fuzzy-based engines design}

Five decision parameters (RSS, BW, NO, CO, and DR) are taken. $\underline{\mathrm{NO}}, \underline{\mathrm{RS}}, \underline{\mathrm{DR}}, \underline{\mathrm{CO}}$, and $\underline{\mathrm{BW}}$ are the fuzzy sets to consider for the respective decision parameter. Four fuzzy memberships (Low, Low-Medium, Medium, Medium-High, and High) are assigned to all the fuzzy sets at the input. Every output of decision depends on the fuzzy rules, which are predefined in the rule base.

$\mu \underline{A Q}(\mathrm{y})=\max _{\mathrm{k}}\left[\min \left[\mu \underline{B W^{\mathrm{k}}}(\right.\right.$ Band Width $), \mu \underline{D R}^{\mathrm{k}}($ Data Rate $\left.\left.)\right]\right]$

for $\mathrm{k}=1,2,3, \ldots, 18$

$\mu \underline{N Q}(\mathrm{y})=\max _{\mathrm{k}}\left[\min \left[\mu \underline{R S}^{\mathrm{k}}(\mathrm{RSS}), \mu \underline{A Q}^{\mathrm{k}}(\mathrm{A}(\right.\right.$ value $\left.\left.))\right]\right]$

for $\mathrm{k}=1,2,3, \ldots, 9$ 
$\mu \underline{M E}(\mathrm{y})=\max _{\mathrm{k}}\left[\min \left[\mu \underline{C O}^{\mathrm{k}}(\right.\right.$ Coverage $), \mu \underline{N O}^{\mathrm{k}}($ Network Occupancy) $\left.\left.)\right]\right]$

for $\mathrm{k}=1,2,3, \ldots, 9$

Defuzzification is done to get a crisp value by converting the aggregated fuzzified data. The A(value), Q(value), M(value) are found by means of the Eqs. (4)-(6) using the centroid method.

$\mathrm{A}_{\text {(value) }}=\frac{\int \mu \underline{A Q}(y) \cdot y d y}{\int \mu \underline{A Q}(y) y d y}$

$\mathrm{Q}_{(\text {value })}=\frac{\int \mu \underline{N Q}(y) \cdot y d y}{\int \mu \underline{N Q}(y) y d y}$

$\mathrm{M}_{(\text {value })}=\frac{\int \mu \underline{M E}(y) \cdot y d y}{\int \mu \underline{M E}(y) y d y}$

The same technique is used in the DS fuzzy engine. Hence, from Eqs. (7) and (8), the aggregated fuzzified data, $\mu \underline{D S}$ and also the crisp output value, $D_{(\text {value })}$ of DS fuzzy engine is obtained.

$\mu \underline{D S}=\max _{\mathrm{k}}\left[\min \left[\mu \underline{D Q}^{\mathrm{k}}(\mathrm{Q}(\right.\right.$ value $)), \mu \underline{D M}^{\mathrm{k}}(\mathrm{M}($ value $\left.\left.))\right]\right]$

for $\mathrm{k}=1,2,3, \ldots, 27$

$\mathrm{D}_{(\text {value })}=\frac{\int \mu \underline{D S}(y) \cdot y d y}{\int \mu \underline{D S}(y) y d y}$

Tabs. 1-3 describe the fuzzy rules of individual fuzzy engine.

Table 1: Fuzzy rules for AQ engine

\begin{tabular}{llll}
\hline Rule No. & Data rate & Bandwidth & Output \\
\hline 1 & Low & Low & Low \\
2 & Low & Medium & Low \\
3 & Low & High & Medium \\
$\ldots$ & & & \\
18 & High & High & High \\
\hline
\end{tabular}

Table 2: Fuzzy rules for NQ engine

\begin{tabular}{llll}
\hline Rule No. & RSS & A $_{\text {(value) }}$ & Output \\
\hline 1 & Low & Low & Low \\
2 & Low & Medium & Low-Medium \\
3 & Low & High & Medium \\
$\ldots$ & & & \\
9 & High & High & High \\
\hline
\end{tabular}


Table 3: Fuzzy rules for DS engine

\begin{tabular}{llll}
\hline Rule No. & $\mathrm{N}_{\text {(value) }}$ & $\mathrm{M}_{\text {(value) }}$ & Output \\
\hline 1 & Low & Low & Low \\
2 & Low & Low & Low-Medium \\
3 & Low & Low & Medium \\
$\ldots$ & & & \\
27 & High & High & High \\
\hline
\end{tabular}

So, based on the value obtained from the DS engine the best network is selected and the handover takes place. For example, if the candidate networks data rate is high and the networks bandwidth is high then the outcome of this rule will be high thereby the A(value) will be high, which is fed as input to the NQ engine.

The Multi-criteria algorithm itself considers a greater number of metrics to select the optimal network. Three different fuzzy engines used in the proposal consider parameters like RSS, data rate, and bandwidth that directly influence the performance of the handover. Hence the parameters packet loss, throughput, and the number of handovers executed were enough for analyzing the performance of the proposed algorithm over the existing state-of-art techniques.

\section{Results and Discussion}

Three various algorithms are considered to choose the finest network. RAMOC, TOPRES, and the Fuzzy-based algorithm are used in the decision making algorithm. These organized algorithms are simulated using a Network simulator-2 tools. To assess the network selection performance, the proposed decision making algorithms that involves different network environments like WLAN, WIFI, WIMAX and UMTS are created. The overall activity of the nodes is monitored by the DH. The movements of the mobile nodes along with the network are monitored in the $\mathrm{DH}$ so that it is easy to trail the movement when the MN enters the environment. The mobile nodes and hetnets are configured as same in the simulation environment. The mobility of mobile nodes is organized by the attaching network. This makes the mobile node attached to the network unaware of the handover process and thereby providing service utilization. Information is passed among mobile nodes and corresponding nodes that are routed by the connected network and the server. The delay of packets is calculated from the time at which the communication is broken and the time at which the communication resumes when it is associated with a new destination network. The network performance is calculated based on various QoS like RSS, data rate, bandwidth, network occupancy, and the coverage.

Based on the coverage of every network, location of the mobility manager is proportional to trail movement of the mobile node. The mobility manager transfers the collected information to the new serving media access gateway (NSMAG) that in turn passes it to the DH. The prejudgement of the interruption of $\mathrm{MN}$ by computing received signal strength, speed and distance of moving node and these are considered by the mobility manager in the message flow. The autonomous distribution values that are frequently generated for each input decision parameter are continuously fed into the decision-making phase which is located in the DH. The simulation runs for a number of times and the average value of the best network is selected from multiple trials obtained. Each algorithm generates the best network as a result. The network which is most often selected by the algorithms is taken into consideration and the handover process is initiated. 


\subsection{Packet Loss}

The proposed model uses the DH, which starts buffering the packets in the Forward Packet Buffer before receiving the HIM from the HSMAG. Upon the MN disconnection from the HSMAG, each transmitted packet is stored in the New Packet Buffer with its sequence number. So, the packet loss is significantly reduced by using two buffers in the double buffer mechanisms. As soon as the proposed algorithms forecast the candidate network for the MN to handoff, the buffered packets are sent to the Mobile Node and the ongoing packets are rerouted to the newly attached network and then to the MN via the NSMAG. The proposed algorithms have less packet loss when compared with existing algorithms [22]. Furthermore, as the NPB in the double buffer mechanism stores the data packets with sequence numbers, the buffered packets will be retransmitted after handover in the exact sequence, and thus, the proposed model solves the issues of out-of-sequence delivery of packets. On utilizing two buffers, packet loss is reduced considerably by up to $1.5 \%$ loss when compared with existing algorithms. Fig. 6 shows the packet loss performance ratio graph using the proposed model.

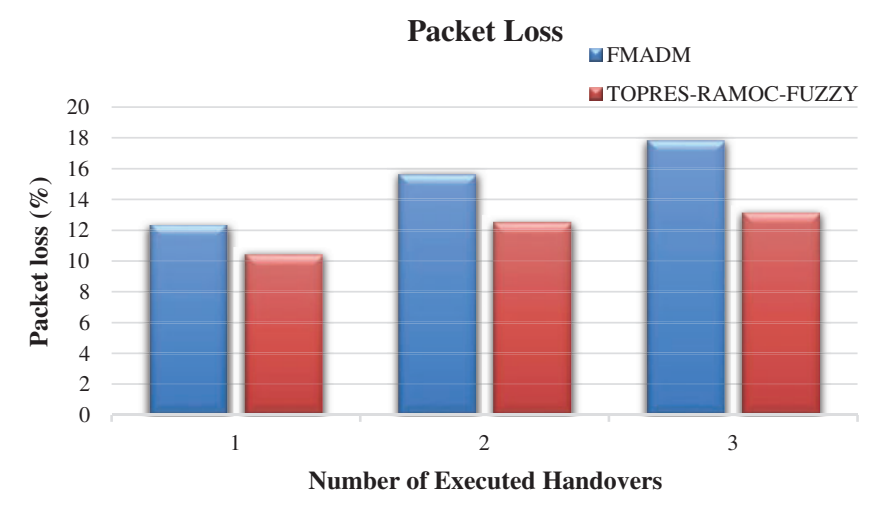

Figure 6: Packet loss (\%) of the proposed algorithm

\subsection{Throughput}

Throughput refers to the total number of data packets that are transferred successfully from the corresponding node to the mobile node in a certain time period. Generally, the transmission of data packets takes place through the $\mathrm{DH}$ and packet buffer with the authentication of the MAG of the network attached. The DH starts evaluating three algorithms when it is intimated that the RSS is weak, and Distance is far, and MN is going to enter an idle state. As the new network for the MN to attach to is predicted by the DH earlier, the throughput is higher, as expected by the user in mobility state. When compared with the existing algorithm [23], the proposed algorithm has high throughput. Three algorithms such as TOPRES, RAMOC and Fuzzy algorithms ensure the increased throughput as a best network is chosen based on maximum results produced individually by these algorithms. The simulation plots shown in Fig. 7 show the throughput obtained in the proposed model.

\subsection{Number of Handovers}

The goodness of the proposed technique is clearly demonstrated as the outcome reduces the number of handovers for selecting the network among all these algorithms when compared with the existing systems. Average number of handovers over each traffic class shown in Fig. 8. 


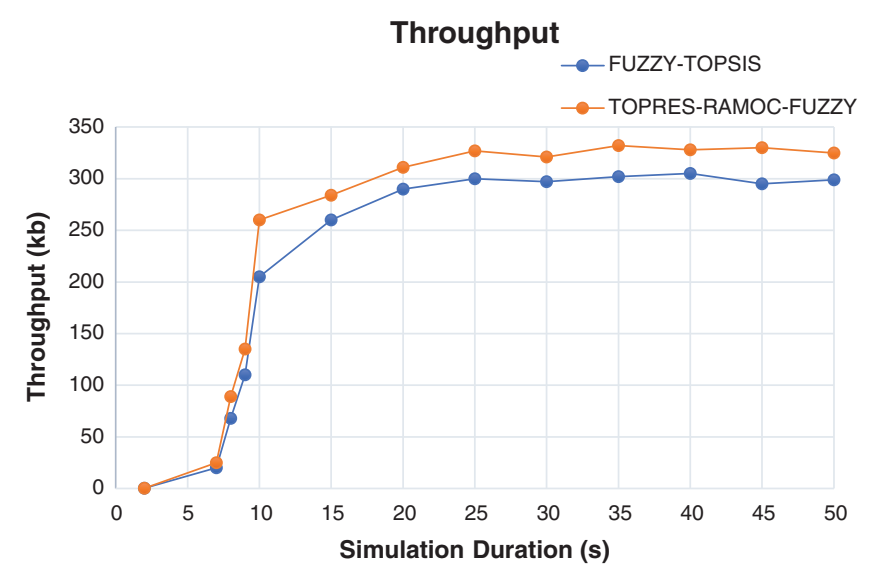

Figure 7: Throughput performance of the proposed algorithm

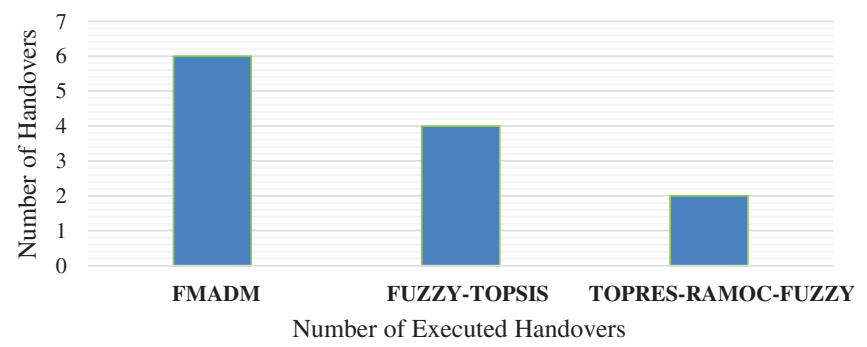

Figure 8: Number of handovers

\section{Conclusion and Future Works}

The problems in the mobility management mechanism and VHO are addressed and these issues are overcome by proposing an intellectual decision making algorithm. By combining multicriteria with fuzzy based decision algorithms, an uninterrupted handover with reduced packet loss and less handover delay is achieved. With various aspects or characteristics, the MN uses algorithms like TOPRES, RAMOC, and formulated fuzzy rules to choose the optimal network. Here, the selection of networks is made based on the result acquired from TOPRES, RAMOC and fuzzy algorithms. The network which produces the optimum result from these algorithms is chosen as the destination network for handover. In addition, in this proposed work the possibility of a mobile node's false handover to the new serving network is calculated, which minimizes the number of false handover. In addition, a double buffering mechanism, which is the usage of two separate buffers, reduces packet loss. The proposed system achieved an enhancement of throughput of up to $10.5 \%$ and a reduction of packet loss of up to $1.5 \%$ to maintain a seamless connection. Further, the number of handovers executed is reduced by up to $50 \%$ when compared to the existing approach and it also avoids the execution of false handover by predicting the best network for handover using a decision making algorithm which combines multi-criteria with fuzzy-based decision making algorithms.

In future works, by reducing the number of input parameters, whose standards are calculated for selecting the best network, VHO algorithms will be optimized. If the numbers of input 
parameters are reduced further, the number of fuzzy rules used in the fuzzy engines to make the handover decision will also be reduced, thereby further reducing the delay in the VHO process.

Acknowledgement: Authors would like to thank the Department of Electrical and Computer Engineering of North South University and Taif University Researchers Supporting Project Number (TURSP-2020/36), Taif University, Saudi Arabia.

Funding Statement: Taif University Researchers Supporting Project Number (TURSP-2020/36), Taif University, Taif, Saudi Arabia.

Conflicts of Interest: The authors declare that they have no conflicts of interest to report regarding the present study.

\section{References}

[1] R. Katru, J. L. M. Iqbal and M. N. Giriprasad, "A survey on vertical handover in heterogeneous networks," International Journal of Innovative Research in Technology, vol. 2, no. 10, pp. 132-136, 2016.

[2] M. D. Jaraiz-Simon, J. A. Gomez-Pulido and M. A. Vega-Rodriguez, "Embedded intelligence for fast QoS-based vertical handoff in heterogeneous wireless access networks," Pervasive and Mobile Computing, vol. 19, no. 1, pp. 141-155, 2015.

[3] A. Prithiviraj, K. Krishnamoorthy and B. Vinothini, "Fuzzy logic based decision making algorithm to optimize the handover performance in HetNets," Circuits and Systems, vol. 7, no. 11, pp. 3756-3777, 2016.

[4] A. Arunachalam, V. Ravi, M. Krichen, R. Alroobaea and J. S. Alqurni, "Analytical comparison of resource search algorithms in non-DHT mobile peer-to-peer networks," Computers, Materials \& Continua, vol. 68, no. 1, pp. 983-1001, 2021.

[5] M. Krichen, O. Cheikhrouhou, M. Lahami, R. Alroobaea and A. Jmal, "Towards a model-based testing framework for the security of internet of things for smart city applications," Lecture Notes of the Institute for Computer Sciences, Social Informatics and Telecommunications Engineering, vol. 224, no. 1, pp. 360-365, 2018.

[6] A. Bhuvaneswari and E. G. D. Prakashraj, "An overview of vertical handoff decision making algorithms," International Journal of Computer Network and Information Security, vol. 9, no. 1, pp. 55-62, 2012.

[7] L. K. Johal and S. Amandeepsingh, "An overview of vertical handover process and techniques," Indian Journal of Science and Technology, vol. 9, no. 14, pp. 1-7, 2016.

[8] J. Santhi and K. Prabha, "An MADM algorithm for vertical handover decision in heterogeneous wireless networks," International Journal of Recent Technology and Engineering, vol. 8, no. 1, pp. 1501-1505, 2019.

[9] G. Mahardhika, M. Ismail and R. Nordin, "Vertical handover decision algorithm using multicriteria metrics in heterogeneous wireless network," Journal of Computer Networks and Communications, vol. 2015, no. 1, pp. 1-8, 2015.

[10] S. Baghla and S. Bansal, "Performance comparison of MADM algorithms for network selection in heterogeneous networks," International Journal on Future Revolution in Computer Science \& Communication Engineering, vol. 4, no. 1, pp. 32-37, 2018.

[11] R. M. Abdullah, A. Z. Abualkishik and A. A. Alwan, "Improved handover decision algorithm using multiple criteria," Proc. EUSPN-2018, vol. 141, no. 1, pp. 32-39, 2018.

[12] R. M. Abdullah and Z. A. Zukarnain, "Vertical handoff algorithm for different wireless technologies," Peer J Preprints5:e2970v1, vol. 1, no. 1, pp. 1-15, 2017.

[13] M. Krichen and R. Alroobaea, "A new model-based framework for testing security of IoT systems in smart cities using attack trees and price timed automata," in Proc. of the 14th Int. Conf. on Evaluation of Novel Approaches to Software Engineering, Portugal, pp. 570-577, 2019. 
[14] M. Sharma, "Fuzzy logic tool for imprecise information in wireless communication-another perspective," International Journal of Research in Management Science \& Technology, vol. 3, no. 1, pp. 57-61, 2015.

[15] J. Jose and A. Prithiviraj, "PMIPV6-HC-MIH: An approach for improving handover performance in NGWN," in 2012 Int. Conf. on Computing, Electronics and Electrical Technologies, Kumaracoil, India, pp. 910-914, 2012.

[16] M. Krichen and R. Alroobaea, "Towards optimizing the placement of security testing components for internet of things architectures," in IEEE/ACS 16th Int. Conf. on Computer Systems and Applications, Abu Dhabi, UAE, pp. 1-2, 2019.

[17] A. Maheswari, A. Prithiviraj and K. Krishnamoorthy, "Markov based VHO to improve the handover performance among heterogeneous wireless networks in PMIPv6 domain," in 2016 Int. Conf. on Circuit, Power and Computing Technologies, Nagercoil, Inida, pp. 1-6, 2016.

[18] M. C. Chuang and J. F. Lee, "DRO: Domain-based route optimization scheme for nested mobile networks," EURASIP Journal on Wireless Communications and Networking, vol. 1, no. 70, pp. 1-19, 2011.

[19] T. M. Lakshmi and V. Prasannavenkatesan, "A comparison of various normalization in techniques for order performance by similarity to ideal solution (TOPSIS)," International Journal of Computing Algorithm, vol. 3, no. 3, pp. 255-259, 2014.

[20] M. Krichen, M. Lahami, O. Cheikhrouhou, R. Alroobaea and A. Jmal, "Security testing of internet of things for smart city applications: a formal approach, smart infrastructure and applications," in EAI/Springer Innovations in Communication and Computing. Cham: Springer, 2020.

[21] T. Piyakul, T. Thanachai, V. Piboonlit and S. Pratit, "Intelligent handover decision based on fuzzy logic for heterogeneous wireless networks," in Proc. ECTI-CON, Hua Hin, Thailand, pp. 1-6, 2015.

[22] V. Ananthanarayanan, A. Rajeswari and V. Sureshkumar, "Service-adaptive fuzzy multi criteria based intelligent vertical handover decision algorithm for heterogeneous wireless networks," Advances in Intelligent Systems and Computing, vol. 324, pp. 297-304, 2015.

[23] I. Chattate, M. El-Khaili and J. Bakkoury, "A new Fuzzy-TOPSIS based algorithm for network selection in next-generation heterogeneous networks," Journal of Communications, vol. 14, no. 3, pp. 194-201, 2019. 